\title{
On the computational modelling of nonlinear electro-elasticity in heterogeneous bodies at finite deformations
}

\author{
Anas Kanan $^{1} \cdot$ Michael Kaliske ${ }^{1}$ \\ Received: 1 September 2020 / Accepted: 9 November 2020/Published online: 27 March 2021 \\ (c) The Author(s) 2021
}

\begin{abstract}
Dielectric elastomer actuators (DEA) have been demonstrated to exhibit a quasi-immediate electro-mechanical actuation response with relatively large deformation capability. The properties of DEA make them suitable to be used in the form of major active components within soft robotics and biomimetic artificial muscles. However, some of the electro-active material properties impose limitations on its applications. Therefore, researchers attempt to modify the structure of the homogeneous DEA material by the incorporation of fillers that possess distinct electro-mechanical properties. This modification of the material's structure leads to a fabricated inhomogeneous composite. From the point of mathematical material modelling and numerical simulation, we propose a material model and a computational framework using the finite element method, which is capable of emulating nonlinear electro-elastic interactions. We consider a coupled electro-mechanical description with the electric and the electro-mechanical properties of the material assumed to be nonlinearly dependent on the deformation. Furthermore, we demonstrate a coupled ansatz that expresses the electric response as dielectrically quasi-linear with only density-dependent electric permittivity. We couple the electro-mechanical models to the extended tube model, which is a suitable approach for the realistic emulation of the hyperelastic response of rubber-like materials. Thereafter, we demonstrate analytical and numerical solutions of a homogeneous electro-elastic body with the Neo-Hookean material model and the extended tube model to express the hyperelastic response. Finally, we use the finite element method to investigate several heterogeneous configurations consisting of soft DEA matrix filled with spherical stiff inclusions with changing volume fraction and ellipsoidal inclusions with varying aspect ratio.
\end{abstract}

Keywords Electro-active polymers · Dielectric elastomer actuators · Electro-mechanical coupling · Finite deformations · Finite element method

\section{Introduction}

Electro-active polymers (EAP) constitute a favorable class of smart materials among others, as their mechanical actuation in response to an electrical stimulus is relatively fast [1]. Moreover, they are capable of exhibiting large strains, where strains up to more than $300 \%$ are observed in some types of EAP [2]. Being motivated by the aforementioned properties, several prototypes of soft artificial muscles and soft robotics have been mainly hinged on EAP; see for example [3-5]. DEA is a

Michael Kaliske

michael.kaliske@tu-dresden.de

Anas Kanan

anas_m_a.kanan@tu-dresden.de

1 Technische Universitat Dresden, Dresden, Germany 
type of EAP that shows electrostrictive behavior [6]. Moreover, it is demonstrated by electro-mechanical experiments of DEA that their coupling electro-mechanical properties can vary depending on deformation [7]. Regarding improving their actuation behavior, introducing an inhomogeneity into DEA through filling its soft matrix with particles that have different properties compared to the soft material constitutes one approach toward an enhancement of the DEA's performance. Several contributions have been devoted to investigate the influence of embedding filler particles in DEA on both the theoretical [8-11] and the practical [11-14] sides, among others. As examples, it is shown in [13, 14] that the actuation behavior of particle-filled DEA gets enhanced as the volume fraction of the fillers increases.

The mathematical description of electro-elastic interactions has been investigated in the last century; see for example [1518]. Relatively recent contributions are proposed to provide detailed mathematical expressions that emulate electro-elasticity considering both materials with deformation-dependent and materials with deformation-independent electro-mechanical properties [19-23]. The implementation of nonlinear electro-elasticity within a finite element framework at the geometrical material setting is described in [24]. Furthermore, the numerical formulation of electro-elasticity in the geometrical spatial setting is demonstrated in [25], where it is pointed out that the implementation of the electro-mechanical finite element in the spatial setting is favorable from a numerical point of view. The influence of the surrounding free space on the electromechanical response of materials is neglected in [24, 25]. In order to take into account the interaction between the material body and the surrounding air, a computational scheme is studied in [26], where the surrounding air is discretized using adaptive meshing.

As the electro-active materials under consideration are rubber-like materials, their response at large strains can be realistically captured by using hyperelastic material models suitable for the simulation of the response of polymers. The model of Arruda and Boyce [27] has been utilized in [28], in order to fit electro-mechanical experiments of DEA. A computational framework of electro-elasticity using the micro-sphere network model is presented in [25]. The Yeoh hyperelastic material model has been employed to predict the response of EAP in [29]. A Gent electro-elastic model is used in [30, 31]. Furthermore, the Mooney-Rivlin model is utilized to describe electro-elasticity in [31].

The nature of the electrical response of materials can be characterized through evaluating the polarization of the material which constitutes the relation between the electric field and the electric displacement. Furthermore, the electro-mechanical coupling of materials can be assessed by examining the relation between the electric field and the mechanical stress or stretch. In the context of mathematical modelling and finite element simulation of electro-mechanical coupling in an EAP structure, the problem can be driven either by an applied electric field or through controlling it by using an electric displacement $[8,9]$, where in turn either the electric displacement or the electric field is considered as the variable describing the electric response, respectively. Furthermore, regarding both aforementioned schemes, the coupled response is expressed by the resulting stretch. Although electro-mechanical stability analysis is not the topic of this paper, it is an aspect that should be pointed out whenever the behavior of DEA is studied. In the context of numerical analysis of stability, the NeoHookean material model coupled to an electro-mechanical ansatz that expresses a quasi-linear dielectric response with only density-dependent electric properties is utilized to simulate inhomogeneous electro-active bodies in [8,9]. Furthermore, it is mentioned in [8] that driving the simulated problem using an electric displacement or surface electric charges is mandatory, in order to simulate the response of EAP structures after they undergo electro-mechanical material or structural instabilities. The latter prerequisite exists due to the fact that the electric field decreases after the instability point is reached; thus, controlling the electric field does not allow the simulation of the response beyond the critical state of the material.

This work is devoted to study the modelling of inhomogeneous electro-active structures, where soft DEA matrices are filled with particles having higher electric permittivity and larger stiffness than the carrier. The influence of varying volume fraction of spherical inclusions and the effect of ellipsoidal inclusions with changing aspect ratio on the electrical and the electro-mechanical overall response of the heterogeneous body are investigated. To this end, we present an electromechanical constitutive model where the hyperelastic material response is expressed by the extended tube model [32] and the electro-mechanical coupling is based on nonlinear electro-elasticity with deformation-dependent electro-mechanical properties of the material, as it is proposed in [21]. In this work, we simplify the problem by neglecting the influence of the surrounding space $[9,24]$. Our main focus in this manuscript is to study the implications of using the electro-elastic model as it is suggested in [21] with regard to the simulation of heterogeneous material structures. Nevertheless, we also demonstrate an electro-mechanical ansatz that expresses the electrical behavior of materials as quasi-linear with only densitydependent electric permittivity [23]. Furthermore, we outline and utilize a monolithically coupled electro-mechanical finite element to perform the numerical analyses. Regarding the simulation examples, we first consider a homogeneous material and demonstrate the analytical solution of the coupled nonlinear electro-elastic model using both the Neo-Hookean and the extended tube model. Moreover, we compare the analytical results to those obtained using the proposed finite element. Thereafter, we demonstrate a series of numerical examples considering material structures with introduced inhomogeneity. 
The manuscript at hand is laid out as follows. Section 2 is devoted to present basic equations that express the balance states of the coupled problem and the corresponding kinematics. In Section 3, a constitutive material model and the linearized terms needed for the corresponding finite element implementation are outlined. In Section 4, an electro-mechanically coupled finite element is described. Section 5 is devoted to present a series of analytical and numerical examples. Finally, the paper is closed with some concluding remarks in Section 6.

\section{General equations of electro-mechanics}

In this section, an overview of the general equations that constitute the basis of coupled electro-mechanics is presented. First, principles of continuum mechanics are used to specifically describe inhomogeneous electro-sensitive materials that are subjected to large deformations. Subsequently, the kinematical link between the mechanical and electrical fields and the associated laws of balance are outlined.

\subsection{Preliminaries}

Considering large deformation, an inhomogeneous material compound $\mathcal{B}$ is split into two different electro-mechanical bodies with distinct material properties, where $\mathcal{B}=\mathcal{B}^{I}+\mathcal{B}^{M}$, as it is shown in Fig. 1. We introduce a nonlinear deformation function $\boldsymbol{x}=\boldsymbol{\varphi}(\boldsymbol{X}, t)$ at time $t \in \mathcal{T}$ that projects points from the material configuration $\boldsymbol{X} \in \mathcal{B}_{0}$ to the spatial setting $\boldsymbol{x} \in \mathcal{B}_{t}$. The deformation gradient $\boldsymbol{F}=\nabla_{\boldsymbol{X}} \boldsymbol{\varphi}$, its cofactor $\operatorname{cof}[\boldsymbol{F}]=\operatorname{det}[\boldsymbol{F}] \boldsymbol{F}^{-T}$, and the Jacobian $J=\operatorname{det}[\boldsymbol{F}]$ map an infinitesimal line, an area element, and a volume element from the material setting to the spatial configuration, respectively. Furthermore, an electric potential $\phi(X, t)$ is defined. The outer boundary $\partial \mathcal{B}=\partial \mathcal{B}^{M}$ is decomposed in terms of the mechanical and the electrical boundary conditions as:

$\partial \mathcal{B}=\partial \mathcal{B}^{\varphi} \cup \partial \mathcal{B}^{t}$, where $\partial \mathcal{B}^{\varphi} \cap \partial \mathcal{B}^{t}=\emptyset \quad$ and $\quad \partial \mathcal{B}=\partial \mathcal{B}^{\phi} \cup \partial \mathcal{B}^{q}$, where $\partial \mathcal{B}^{\phi} \cap \partial \mathcal{B}^{q}=\emptyset$,

respectively. The portion $\partial \mathcal{B}^{\varphi}$ denotes the Dirichlet mechanical boundary and $\partial \mathcal{B}^{\phi}$ constitutes the electrical counterpart. Furthermore, the Neumann boundaries read $\partial \mathcal{B}^{t}$ and $\partial \mathcal{B}^{q}$, where mechanical tractions and surface charges can be prescribed, respectively.

\subsection{Electro-mechanics of deformable continua}

The electrical part of the coupled problem is based on two Maxwell equations: the Gauss law for electricity and Faraday's law. In the absence of varying magnetic fields, the associated Maxwell equations can be introduced in terms of the electric displacement $\mathbb{d}$ and the electric field $\mathbb{e}$ in the spatial setting, as

$\nabla_{\boldsymbol{x}} \cdot \mathbb{d}=\varrho_{t} \quad$ and $\quad \nabla_{\boldsymbol{x}} \times \mathbb{e}=\mathbf{0}$ in $\mathcal{B}_{t}$,

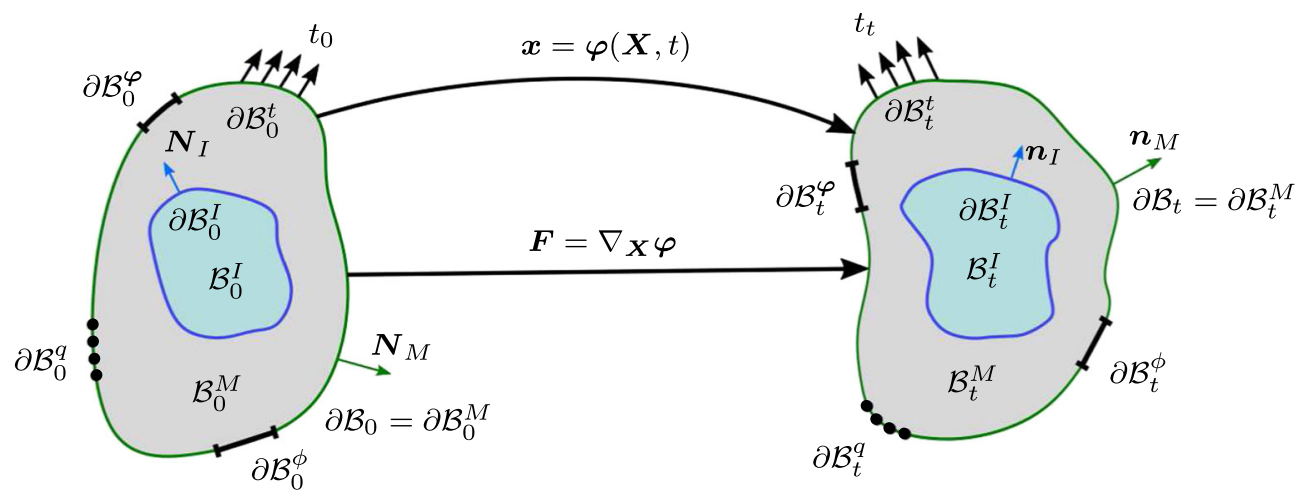

Fig. 1 Nonlinear mapping from the undeformed to the deformed configuration of an inhomogeneous electro-mechanical body 
respectively. The operator $\nabla_{\boldsymbol{x}} \cdot[\bullet]$ reads the divergence with respect to the spatial coordinates $\boldsymbol{x}$ and $\nabla_{\boldsymbol{x}} \times[\bullet]$ is the curl of a vector at the deformed setting. Moreover, $\varrho_{t}$ denotes prescribed free charges per unit current volume [10]. Equation (2.2) enables expressing the spatial electric field $\mathbb{e}$ as the negative gradient of a scalar electric potential $\phi$, where

$\mathbb{e}=-\nabla_{x} \phi$ in $\mathcal{B}_{t}$.

Regarding the electrical state at the interface $\partial \mathcal{B}^{I}$, the jump conditions

$\llbracket \mathbb{d} \rrbracket \cdot \boldsymbol{n}_{I}=q_{I} \quad$ and $\quad \llbracket \mathbb{e} \rrbracket \times \boldsymbol{n}_{I}=\mathbf{0} \quad$ on $\quad \partial \mathcal{B}_{t}^{I}$

apply [33], where $\llbracket \bullet \rrbracket:=[\bullet]^{\text {out }}-[\bullet]^{\text {in }}$ and $q_{I}$ denotes the density of free surface charges at the interface $\partial \mathcal{B}^{I}$. Due to the fact that the influence of the surrounding space is neglected in this contribution, the electrical conditions at the outer boundary $\partial \mathcal{B}^{M}=\partial \mathcal{B}$ can be expressed as

$\mathbb{d} \cdot \boldsymbol{n}_{M}=-q_{M} \quad$ on $\quad \partial \mathcal{B}_{t}^{q} \quad$ and $\quad \mathbb{e} \times \boldsymbol{n}_{M}=\mathbf{0} \quad$ on $\quad \partial \mathcal{B}_{t}^{\phi}$,

with the density of free charges $q_{M}$ at the outer boundary $\partial \mathcal{B}_{t}^{q}$. Regarding the continuity of the scalar electric potential $\phi$ on the interface $\partial \mathcal{B}^{I}$, the following condition applies:

$\llbracket \phi \rrbracket=0 \quad$ on $\quad \partial \mathcal{B}_{t}^{I}$.

Moreover, a scalar electric potential $\phi=\bar{\phi}$ can be prescribed on the outer Dirichlet boundary $\partial \mathcal{B}^{\phi}$. The electrical polarization of materials is expressed mathematically through the generalized relation:

$\mathbb{d}=\epsilon_{0} \mathbb{e}+p$ in $\mathcal{B}_{t}$,

with the contribution $\epsilon_{0} \mathbb{e}$ as the electric displacement in vacuum and $p$ as the polarization of deformable matter at the current configuration. In the free space, the relation $d=\epsilon_{0} \mathbb{e}$ applies, with the constant electric permittivity of the vacuum $\left(\epsilon_{0}=8.854 \times 10^{-12} \mathrm{~N} / \mathrm{V}^{2}\right)$, which in turn expresses the electric response in free space as linear. On the other hand, the relation between the polarization $p$ and the electric field $\mathbb{e}$ can take a nonlinear form. The electric field, electric displacement, and polarization at the reference configuration are linked to their spatial counterparts, through the pull-backs:

$\mathbb{E}=\boldsymbol{F}^{T} \mathbb{e}, \quad \mathbb{D}=\mathbb{d} \operatorname{cof}[\boldsymbol{F}] \quad$ and $\quad \mathbb{P}=\mathbb{p} \operatorname{cof}[\boldsymbol{F}] \quad$ in $\mathcal{B}_{0}$,

respectively. As deformation measures, we introduce the right and the left Cauchy-Green tensors as

$\boldsymbol{C}=\boldsymbol{F}^{T} \boldsymbol{g} \boldsymbol{F} \quad$ and $\quad \boldsymbol{b}=\boldsymbol{F} \boldsymbol{G}^{-1} \boldsymbol{F}^{T}$

where $\boldsymbol{g}$ is the Eulerian metric tensor and $\boldsymbol{G}$ denotes the Lagrangian metric tensor. Both tensors $\boldsymbol{g}$ and $\boldsymbol{G}$ are covariant and symmetric. The balance of linear momentum is written in terms of the total Cauchy stresses $\sigma$, with neglecting the inertial effects as

$\nabla_{\boldsymbol{x}} \cdot \boldsymbol{\sigma}+\rho \boldsymbol{\gamma}^{m e c}=\mathbf{0}$ in $\mathcal{B}_{t}$,

with the volume-specific mechanical body forces $\rho \gamma$ at the spatial setting. In the context of coupled electro-mechanics, the total stress tensor $\sigma$ includes a contribution that is originated due to electric body forces [19, 34]. The total first Piola-Kirchhoff stress tensor $\boldsymbol{P}$ can be expressed as a pull-back of the total Cauchy stress tensor $\boldsymbol{\sigma}$, where

$\boldsymbol{P}=\boldsymbol{\sigma} \operatorname{cof}[\boldsymbol{F}]$ in $\mathcal{B}_{0}$.

The Dirichlet and Neumann mechanical jump conditions at the interface $\partial \mathcal{B}^{I}$ can be expressed as

$\llbracket \boldsymbol{\varphi} \rrbracket=\mathbf{0} \quad$ and $\quad \llbracket \boldsymbol{\sigma} \rrbracket \cdot \boldsymbol{n}_{I}=-\boldsymbol{t}_{I} \quad$ on $\quad \partial \mathcal{B}_{t}^{I}$,

respectively, with the traction $\boldsymbol{t}_{I}$ at the boundary $\partial \mathcal{B}_{t}^{I}$. Moreover, mechanical boundary conditions can be prescribed on the outer boundary $\partial \mathcal{B}^{M}=\partial \mathcal{B}$ as

$\boldsymbol{\varphi}=\overline{\boldsymbol{\varphi}} \quad$ on $\quad \partial \mathcal{B}_{t}^{\varphi} \quad$ and $\quad \boldsymbol{\sigma} \cdot \boldsymbol{n}_{M}=\boldsymbol{t}_{M} \quad$ on $\quad \partial \mathcal{B}_{t}^{t}$. 


\section{Constitutive model for electro-elasticity}

This section is devoted to demonstrate a material model that describes the coupled electro-elastic behavior of EAP at large strains. As a first step, we introduce the constitutive equations and the terms needed for the finite element implementation in their general form. Subsequently, we present the material model and the associated algorithmic expressions.

\subsection{Generalized constitutive description and material-tangent moduli}

The coupled material response is expressed by introducing a total energy-enthalpy density function $\hat{\Psi}^{\text {tot }}$, which is decomposed into a purely hyperelastic energy contribution $\Psi^{\text {hypel }}$ and an electro-elastic enthalpy density $\bar{\Psi}^{\text {coup }}$ as

$\hat{\Psi}^{\text {tot }}(\boldsymbol{F}, \boldsymbol{g}, \mathbb{E})=\Psi^{\text {hypel }}(\boldsymbol{F}, \boldsymbol{g})+\bar{\Psi}^{\text {coup }}(\boldsymbol{F}, \boldsymbol{g}, \mathbb{E})$.

We introduce the multiplicative decomposition of the total deformation gradient $\boldsymbol{F}$ into a volumetric $\boldsymbol{F}^{v o l}$ and an isochoric contribution $\overline{\boldsymbol{F}}$ as follows:

$\boldsymbol{F}=\boldsymbol{F}^{v o l} \overline{\boldsymbol{F}}, \quad$ with $\quad \boldsymbol{F}^{v o l}=J^{1 / 3} \mathbf{1} \quad$ and $\quad \overline{\boldsymbol{F}}=J^{-1 / 3} \boldsymbol{F}$,

where 1 reads the second-order identity tensor. Based on the volumetric-isochoric split of the deformation in Eq. 15, we define an additive split of the hyperelastic energy contribution $\Psi^{\text {hypel }}$ into

$\Psi^{\text {hypel }}(\boldsymbol{F}, \boldsymbol{g})=\Psi^{\text {vol }}(J)+\Psi^{i s o}(\overline{\boldsymbol{F}}, \boldsymbol{g})$,

where $\Psi^{v o l}$ denotes a purely volumetric part and $\Psi^{i s o}$ constitutes a volume-preserving contribution of the energy density. Referring to the decompositions of the total energy-enthalpy density function as it is shown in Eqs. 14 and 16, the total Cauchy stresses $\sigma$ can be decomposed and introduced as

$\boldsymbol{\sigma}=\boldsymbol{\sigma}^{\text {vol }}+\boldsymbol{\sigma}^{i s o}+\boldsymbol{\sigma}^{\text {coup }}=2 J^{-1}\left(\partial_{\boldsymbol{g}} \Psi^{\text {vol }}(J)+\partial_{\boldsymbol{g}} \Psi^{i s o}(\overline{\boldsymbol{F}}, \boldsymbol{g})+\partial_{g} \bar{\Psi}^{\text {coup }}(\boldsymbol{F}, \boldsymbol{g}, \mathbb{E})\right)$.

The electric displacement vector $d$ at the current configuration is given as

$\mathbb{d}=-J^{-1} \partial_{\mathbb{e}} \bar{\Psi}^{\text {coup }}(\boldsymbol{F}, \boldsymbol{g}, \mathbb{E})$.

In order to implement the constitutive model within a fully monolithic finite element framework, we introduce the material-tangent moduli as follows:

$\mathbb{C} \varphi \varphi=4 J^{-1} \partial^{2}{ }_{\boldsymbol{g} g} \hat{\Psi}^{\text {tot }}(\boldsymbol{F}, \boldsymbol{g}, \mathbb{E}) \quad, \quad \mathbf{C} \boldsymbol{\varphi} \phi=-2 J^{-1} \partial^{2}{ }_{\boldsymbol{g} \mathbb{\Theta}} \bar{\Psi}^{\operatorname{coup}}(\boldsymbol{F}, \boldsymbol{g}, \mathbb{E})$,

$\mathbf{C} \phi \boldsymbol{\varphi}=-2 J^{-1} \partial^{2}{ }_{\mathrm{eg}} \bar{\Psi}^{\text {coup }}(\boldsymbol{F}, \boldsymbol{g}, \mathbb{E}), \quad \mathbf{C} \phi \phi=J^{-1} \partial^{2}{ }_{\mathfrak{e e}} \bar{\Psi}^{\text {coup }}(\boldsymbol{F}, \boldsymbol{g}, \mathbb{E})$,

with the fourth-order tensor $\mathbb{C} \varphi \varphi$, the third-order coupled material tensors $\mathbf{C} \varphi \phi$ and $\mathbf{C} \phi \varphi$. Moreover, $\mathbf{C} \phi \phi$ denotes the second-order material tensor. The associated Lie derivatives of the coupled problem are shown in [25]. According to the splits of the total energy-enthalpy density function as demonstrated in Eqs. 14 and 16, the decomposition of the fourth-order material tensor $\mathbb{C} \varphi \varphi$ takes the form:

$\mathbb{C} \varphi \varphi=\mathbb{C} \varphi \varphi^{\text {vol }}+\mathbb{C} \varphi \varphi^{i s o}+\mathbb{C} \varphi \varphi^{\text {coup }}$,

with the volumetric part $\mathbb{C} \varphi \varphi^{\text {vol }}$, the isochoric contribution $\mathbb{C} \varphi \varphi^{i s o}$, and the coupled part $\mathbb{C} \varphi \varphi^{\text {coup }}$.

\subsection{Hyperelastic material model}

The description of the hyperelastic response of the material is achieved by specifying the volumetric part $\Psi^{v o l}$ and the isochoric part $\Psi^{i s o}$ of the total energy-enthalpy function. The contribution $\Psi^{v o l}$ is chosen in a way that allows the simulation of both compressible and quasi-incompressible materials, and is introduced as:

$\Psi^{v o l}(J)=\frac{\kappa}{4}\left(J^{2}-2 \ln J-1\right)$, 
where $\kappa$ is the bulk modulus. The volumetric Cauchy stresses $\sigma^{v o l}$ and the associated moduli $\mathbb{C} \varphi \varphi^{\text {vol }}$ can be computed as:

$$
\begin{aligned}
& p=\partial_{J} \Psi_{e}^{v o l}(J)=\frac{\kappa}{2}\left(\frac{J^{2}-1}{J}\right), \\
& s=J \partial_{J J}^{2} \Psi_{e}^{v o l}(J)=\frac{\kappa}{2}\left(\frac{J^{2}+1}{J}\right), \\
& \sigma^{v o l}=p g^{-1}, \\
& \mathbb{C} \varphi \varphi^{v o l}=(p+s) g^{-1} \otimes g^{-1}-2 p \square^{g^{-1}},
\end{aligned}
$$

with the fourth-order identity tensor $\square^{\boldsymbol{A}}{ }_{i j k l}=\left(A_{i k} A_{j l}+A_{i l} A_{j k}\right) / 2$, where the second-order arbitrary tensor $\boldsymbol{A}$ is defined as $\boldsymbol{A}=A_{i j} \mathbf{e}_{i} \otimes \mathbf{e}_{j}$ in terms of the Cartesian basis $\mathbf{e}_{i}$. The extended-tube model [32] is utilized to describe the isochoric part $\Psi^{i s o}$ of the total energy density function. Taking into regard the limited chain extensibility of the network chains and the topological tube constraints, the isochoric contribution $\Psi^{i s o}$ is split into a cross-linking contribution $W^{c}$ and a topological tube constraints part $L^{e}$, where

$$
\begin{aligned}
& \Psi^{i \text { so }}(\overline{\boldsymbol{C}})=W^{c}\left(\bar{I}_{\overline{\boldsymbol{C}}}\right)+L^{e}\left(\bar{\lambda}_{a}\right), \\
& W^{c}\left(\bar{I}_{\overline{\boldsymbol{C}}}\right)=\frac{G_{c}}{2}\left[\frac{\left(1-\delta^{2}\right)\left(\bar{I}_{\overline{\boldsymbol{C}}}-3\right)}{1-\delta^{2}\left(\bar{I}_{\overline{\boldsymbol{C}}}-3\right)}+\ln \left(1-\delta^{2}\left(\bar{I}_{\overline{\boldsymbol{C}}}-3\right)\right)\right], \\
& L^{e}\left(\bar{\lambda}^{a}\right)=2 \frac{G_{e}}{\beta^{2}} \sum_{a=1}^{3}\left(\bar{\lambda}_{a}^{-\beta}-1\right),
\end{aligned}
$$

with the cross-linking $G_{c}$ and the topological constraints $G_{e}$ shear moduli. Limited chain extensibility is expressed by the material parameter $\delta$ and the topological constraints are taken into account through the parameter $\beta$. Moreover, $\bar{I}_{\overline{\boldsymbol{C}}}=\operatorname{tr} \overline{\boldsymbol{C}}$ reads the first invariant of the isochoric right Cauchy-Green tensor, with $\bar{C}=J^{-2 / 3} \boldsymbol{C}$. For the computation of the isochoric stresses $\sigma^{i s o}$ and tangent moduli $\mathbb{C} \varphi \varphi^{i s o}$, we refer to $[35,36]$.

\subsection{Dielectrically quasi-linear material response}

The electric response of the material can expressed as quasi-linear regarding the relation between the current electric field $\mathbb{e}$ and the current electric displacement $\mathbb{d}$ with density-dependent electric permittivity, by specifying $\bar{\Psi}^{\text {coup }}$ as

$\bar{\Psi}^{\operatorname{coup}}(\boldsymbol{C}, \mathbb{E})=-\frac{1}{2} \epsilon \boldsymbol{C}^{-1}:[\mathbb{E} \otimes \mathbb{E}]$

where $\epsilon=\epsilon_{m} \epsilon_{0}$ reads the electric permittivity of the material, with $\epsilon_{m}$ as the associated dielectric constant. Referring to the definition in Eq. 18, the electric displacement $\mathbb{d}$ takes the form:

$\mathbb{d}=\hat{\epsilon}(J) \boldsymbol{g}^{-1} \mathbb{e}$,

with the density-dependent permittivity $\hat{\epsilon}(J)=J^{-1} \epsilon$. The coupled stress $\sigma^{\text {coup }}$ can be expressed as

$\sigma^{\text {coup }}=\hat{\epsilon}(J) \rrbracket^{g^{-1}}:[\mathbb{e} \otimes \mathbb{e}]$.

Consequently, using Eq. 19, the associated material-tangent terms are introduced as

$$
\begin{aligned}
& \mathbb{C} \varphi \varphi^{\text {coup }}=-4 \hat{\epsilon}(J)\left[\rrbracket^{g^{-1}} \otimes g^{-1}\right]:[\mathbb{e} \otimes \mathbb{e}] \\
& \mathbf{C} \varphi \phi^{\text {coup }}=-2 \hat{\epsilon}(J) \rrbracket^{g^{-1}} \mathbb{e} \\
& \mathbf{C} \phi \phi^{\text {coup }}=-\hat{\epsilon}(J) g^{-1} .
\end{aligned}
$$




\subsection{Nonlinear electric response}

Referring to [21], we describe electro-elasticity with a nonlinear deformation-dependent electrical and electro-mechanical response of the material by introducing the invariants

$I_{4}=\boldsymbol{G}^{-1}:[\mathbb{E} \otimes \mathbb{E}], \quad I_{5}=\boldsymbol{C}:[\mathbb{E} \otimes \mathbb{E}], \quad I_{6}=\boldsymbol{C}^{2}:[\mathbb{E} \otimes \mathbb{E}]$,

and setting the coupled enthalpy function $\bar{\Psi}^{\text {coup }}$ as

$\bar{\Psi}^{\text {coup }}(\boldsymbol{C}, \mathbb{E})=c_{1} I_{4}+c_{2} I_{5}$,

with the material parameter $c_{1}$ that has an influence on the polarization of the material and the parameter $c_{2}$ which influences the polarization of the material and describes the corresponding electro-mechanical coupling. Consequently, the electric induction can be introduced as

$\mathbb{d}^{\text {coup }}=\left(-2 \hat{c}_{1}(J) \boldsymbol{b}-2 \hat{c}_{2}(J) \boldsymbol{F} \cdot \boldsymbol{C} \cdot \boldsymbol{F}^{T}\right) \mathbb{e}$,

where $\hat{c}_{1}(J)=J^{-1} c_{1}$ and $\hat{c}_{2}(J)=J^{-1} c_{2}$. In what follows of this section, we set $\boldsymbol{g}=\mathbf{1}$ which is the case for Cartesian coordinates. Regarding the electro-mechanical coupling, we introduce the stresses $\sigma^{\text {coup }}$ as

$\boldsymbol{\sigma}^{\text {coup }}=2 \hat{c}_{2}(J) \boldsymbol{b} \mathbb{e} \otimes \boldsymbol{b} \mathbb{e}$.

For the sake of completeness with regard to the finite element implementation of the model, we refer to Eq. 19 and compute the tangential material tensors as:

$\mathbb{C} \varphi \varphi^{\text {coup }}=\mathbf{0}$,

$\mathbf{C} \varphi \phi^{\text {coup }}=-4 c_{2} \square^{b}$,

$\mathbf{C} \phi \phi^{\text {coup }}=2 c_{1} \boldsymbol{b}+2 c_{2} \boldsymbol{b}^{2}$.

\section{Finite element implementation}

In this section, we first explain the weak forms of the main governing equations for the coupled electro-mechanical problem under consideration. Thereafter, in the context of a fully monolithic finite element, the linearizations of the weak forms are presented. In a last step, we outline the discretization procedure of the introduced finite element approach.

\subsection{Weak formulation and its linearization}

Following the method of weighted residuals, we multiply the strong forms in Eqs. 2.1 and 10 by the virtual test functions $\delta \varphi$ and $\delta \phi$, where both test functions vanish at the boundaries $\partial \mathcal{B}^{\varphi}$ and $\partial \mathcal{B}^{\phi}$, respectively. Subsequently, an integration by parts is applied and the Gauss theorem is used, in order to introduce the weak forms of Eqs. 10 and 2.1 as

$G^{\varphi}(\delta \varphi, \varphi, \phi)=\int_{\mathcal{B}} \nabla_{x} \delta \varphi: \sigma d v-\int_{\partial \mathcal{B}} \delta \varphi \cdot t d a=0$,

$G^{\phi}(\delta \phi, \phi, \varphi)=\int_{\mathcal{B}} \nabla_{x} \delta \phi \cdot d d v+\int_{\partial \mathcal{B}} \delta \phi q d a=0$,

respectively, where the terms associated with the free volume charges $\varrho_{t}$ and the mechanical body forces $\rho \boldsymbol{\gamma}$ are not demonstrated. Regarding (33), in the following, we only consider the treatment of the terms within the solid domain $\mathcal{B}$. As a first step toward the consistent linearization of the coupled problem, we define the increments $\Delta \varphi$ and $\Delta \phi$ that correspond to the main state variables $\varphi$ and $\phi$, respectively. Subsequently, we utilize the directional derivative of the weak form expression in Eq. 33.1 along $\Delta \varphi$ and its counterpart in Eq. 33.2 along $\Delta \phi$, to introduce the linearized forms:

$$
\begin{aligned}
& L\left[G^{\varphi}\right](\delta \varphi, \varphi, \phi, \Delta \varphi, \Delta \phi)=G^{\varphi}(\delta \varphi, \varphi, \phi)+\Delta G^{\varphi}(\delta \varphi, \varphi, \phi, \Delta \varphi, \Delta \phi)=0, \\
& L\left[G^{\phi}\right](\delta \phi, \varphi, \phi, \Delta \varphi, \Delta \phi)=G^{\phi}(\delta \phi, \varphi, \phi)+\Delta G^{\phi}(\delta \phi, \varphi, \phi, \Delta \varphi, \Delta \phi)=0,
\end{aligned}
$$


with the expressions

$\Delta G^{\varphi}(\delta \varphi, \varphi, \phi, \Delta \varphi, \Delta \phi)=D\left[G^{\varphi}\right](\delta \varphi, \varphi, \phi) \cdot \Delta \varphi+D\left[G^{\varphi}\right](\delta \varphi, \varphi, \phi) \Delta \phi$,

$\Delta G^{\phi}(\delta \phi, \varphi, \phi) \quad=D\left[G^{\phi}\right](\delta \phi, \varphi, \phi) \cdot \Delta \varphi+D\left[G^{\phi}\right](\delta \phi, \varphi, \phi) \Delta \phi$,

where $L$ reads the linearization and $D$ denotes the directional derivative of the residual terms. The identities $\nabla_{x} \delta \varphi=$ $\nabla_{\boldsymbol{X}} \delta \boldsymbol{\varphi} \boldsymbol{F}^{-1}$ and $\Delta \boldsymbol{F}^{-1}=-\boldsymbol{F}^{-1} \nabla_{x} \Delta \boldsymbol{\varphi}$ are utilized to express the incremental form of the gradient term $\nabla_{\boldsymbol{x}} \delta \boldsymbol{\varphi}$ as

$\Delta \nabla_{x} \delta \varphi=-\nabla_{x} \delta \varphi \nabla_{x} \Delta \varphi$

Consequently, the linearized form of the total Cauchy stresses $\sigma$ is expressed as

$\Delta \sigma=\mathbb{C} \varphi \varphi: g \nabla_{x} \Delta \varphi+\nabla_{x} \Delta \varphi \sigma+\sigma\left(\nabla_{x} \Delta \varphi\right)^{T}+\mathbf{C} \varphi \phi \nabla_{x} \Delta \phi$,

with the the fourth-order $\mathbb{C} \varphi \varphi$ and third-order $\mathbf{C} \varphi \phi$ material moduli, as they are defined in Eq. 19. Note that based on the material model presented in Section 3, the symmetries $\mathbb{C} \varphi \varphi_{i j k l}=\mathbb{C} \varphi \varphi_{k l i j}, \mathbb{C} \varphi \varphi_{i j k l}=\mathbb{C} \varphi \varphi_{j i l k}$, and $\mathbf{C} \varphi \phi_{i j k}=\mathbf{C} \varphi \phi_{j i k}$ apply. The relations $\nabla_{x} \delta \phi=\nabla_{\boldsymbol{X}} \delta \phi \boldsymbol{F}^{-1}$ and $\Delta \boldsymbol{F}^{-1}=-\boldsymbol{F}^{-1} \nabla_{\boldsymbol{x}} \Delta \boldsymbol{\varphi}$ are exploited to describe the incremental expression of the gradient term $\nabla_{x} \delta \phi$ as

$\Delta \nabla_{x} \delta \phi=-\nabla_{x} \delta \phi \nabla_{x} \Delta \varphi$

Thereafter, linearization of the electric displacement $\mathbb{d}$ can be evaluated as

$\Delta \mathbb{d}=\boldsymbol{C} \phi \boldsymbol{\varphi}: \boldsymbol{g} \nabla_{\boldsymbol{x}} \Delta \boldsymbol{\varphi}+\nabla_{\boldsymbol{x}} \Delta \boldsymbol{\varphi} \mathbb{d}+\mathbf{C} \phi \phi \nabla_{\boldsymbol{x}} \Delta \phi$

with $\mathbf{C} \phi \varphi$ and $\mathbf{C} \phi \phi$ as introduced in Eq. 19. As the linearized terms that exist within the solid domain $\mathcal{B}$, as shown in Eqs. 33.1 and 33.2, are obtained, we can express (35.1) and (35.2) as:

$$
\Delta G^{\varphi}=\int_{\mathcal{B}} \nabla_{x} \delta \varphi: \nabla_{x} \Delta \varphi \sigma d v+\int_{\mathcal{B}} \nabla_{x} \delta \varphi: \mathbb{C} \varphi \varphi: g \nabla_{x} \Delta \varphi d v+\int_{\mathcal{B}} \nabla_{x} \delta \varphi: \mathbf{C} \varphi \phi \cdot \nabla_{x} \Delta \phi d v
$$

and

$$
\Delta G^{\phi}=\int_{\mathcal{B}} \nabla_{x} \delta \phi \cdot \mathbf{C} \phi \varphi: g \nabla_{x} \Delta \varphi d v+\int_{\mathcal{B}} \nabla_{x} \delta \phi \cdot \mathbf{C} \phi \phi \cdot \nabla_{x} \Delta \phi d v
$$

respectively.

\subsection{Finite element discretization}

The finite element method is employed to discretize (33) and (40) by decomposing the domain $\mathcal{B}$ into solid elements, where $\mathcal{B} \approx \bigcup_{e=1}^{n_{e}} \mathcal{B}^{e}$, with the total number of finite elements $n_{e}$. Following the isoparametric concept of the finite element method, the deformation field $\varphi^{e}$, the electric potential $\phi^{e}$, and the corresponding virtual functions are interpolated over the element $\mathcal{B}^{e}$ using nodal values and shape functions, where:

$$
\begin{aligned}
\varphi^{e} & =\sum_{I=1}^{n_{e n}} N^{I} \varphi^{I}, & \delta \varphi^{e} & =\sum_{I=1}^{n_{e n}} N^{I} \delta \varphi^{I}, \\
\phi^{e} & =\sum_{I=1}^{n_{e n}} N^{I} \phi^{I}, & \delta \phi^{e} & =\sum_{I=I}^{n_{e n}} N^{I} \delta \phi^{I},
\end{aligned}
$$


with the number of the finite element nodes $n_{e n}$. In analogy to Eq. 41, we discretize the gradients of the increments $\Delta \varphi^{e}$ and $\Delta \phi^{e}$ and the gradients of the test functions $\delta \varphi^{e}$ and $\delta \phi^{e}$ as

$$
\begin{array}{ll}
\nabla_{\boldsymbol{x}} \Delta \varphi^{e}=\sum_{I=1}^{n_{e n}} \Delta \varphi^{I} \otimes \nabla_{x} N^{I}, & \nabla_{\boldsymbol{x}} \delta \varphi^{e}=\sum_{I=1}^{n_{e n}} \delta \varphi^{I} \otimes \nabla_{\boldsymbol{x}} N^{I}, \\
\nabla_{\boldsymbol{x}} \Delta \phi^{e}=\sum_{I=1}^{n_{e n}} \Delta \phi^{I} \otimes \nabla_{\boldsymbol{x}} N^{I}, & \nabla_{\boldsymbol{x}} \delta \phi^{e}=\sum_{I=1}^{n_{e n}} \delta \phi^{I} \otimes \nabla_{\boldsymbol{x}} N^{I} .
\end{array}
$$

A discretized form of Eq. 33 can be defined by using the interpolated expressions in Eqs. 41 and 42 as

$\mathbb{R}=\mathrm{A}_{e=1}^{n_{e}} \mathbb{R}_{\mathrm{el}}=\mathbf{0}$ with $\mathbb{R}_{\mathrm{el}}=\left\{\begin{array}{l}\mathbb{R}^{\varphi} \\ \mathbb{R}^{\phi}\end{array}\right\}$,

where

$$
\begin{aligned}
& \mathbb{R}^{\varphi}=\int_{\mathcal{B}^{e}} \nabla_{\boldsymbol{x}} N^{I} \cdot \sigma d v, \\
& \mathbb{R}^{\phi}=\int_{\mathcal{B}^{e}} \nabla_{\boldsymbol{x}} N^{I} \cdot d d v,
\end{aligned}
$$

with the global residual vector $\mathbb{R}$ and the operator $A$ that represents the global assembly of $\mathbb{R}_{\mathrm{el}}$ evaluated at the local nodes, based on the element connectivity. Similarly, we discretize (40) and introduce the global tangent matrix $\mathbb{K}$ as

$\mathbb{K}=\mathrm{A}_{e=1}^{n_{e}} \mathbb{K}_{\mathrm{el}} \quad$ with $\quad \mathbb{K}_{\mathrm{el}}:=\left[\begin{array}{ll}\mathbb{K}^{\varphi \varphi} & \mathbb{K}^{\varphi \phi} \\ \mathbb{K}^{\phi \varphi} & \mathbb{K}^{\phi \phi}\end{array}\right]$,

where

$$
\begin{aligned}
\mathbb{K}^{\varphi \varphi} & =\int_{\mathcal{B}^{e}} \nabla_{x} N^{I} \cdot \boldsymbol{\sigma} \cdot \nabla_{x} N^{J} d v+\int_{\mathcal{B}^{e}} \nabla_{\boldsymbol{x}} N^{I} \cdot \mathbb{C} \boldsymbol{\varphi} \boldsymbol{\theta} \cdot \nabla_{\boldsymbol{x}} N^{J} d v, \\
\mathbb{K}^{\varphi \phi} & =\int_{\mathcal{B}^{e}} \nabla_{\boldsymbol{x}} N^{I} \cdot \mathbf{C} \boldsymbol{\varphi} \phi \cdot \nabla_{\boldsymbol{x}} N^{J} d v, \\
\mathbb{K}^{\phi \varphi} & =\int_{\mathcal{B}^{e}} \nabla_{\boldsymbol{x}} N^{I} \cdot \mathbf{C} \phi \boldsymbol{\varphi} \cdot \nabla_{\boldsymbol{x}} N^{J} d v=\int_{\mathcal{B}^{e}} \nabla_{\boldsymbol{x}} N^{J} \cdot \mathbf{C} \boldsymbol{\varphi} \phi \cdot \nabla_{\boldsymbol{x}} N^{I} d v, \\
\mathbb{K}^{\phi \phi} & =\int_{\mathcal{B}^{e}} \nabla_{\boldsymbol{x}} N^{I} \cdot \mathbf{C} \phi \phi \cdot \nabla_{\boldsymbol{x}} N^{J} d v,
\end{aligned}
$$

with the element stiffness matrix $\mathbb{K}_{\mathrm{el}}$.

\section{Numerical examples}

This section is devoted to demonstrate the features of the presented material model within a finite element framework. Examples of both homogeneous and inhomogeneous electro-mechanical bodies are depicted. For all the considered electromechanical simulations, an electric field develops due to the application of a voltage difference between two electrodes that cover the surfaces of the body under consideration. However, the influence of the electrodes is neglected, assuming that their thickness is relatively small compared to the thickness of the body, which is usually the case. For all the finite element analyses, we use four-noded displacement-based tetrahedral finite elements. The first example is meant to demonstrate an analytical solution of the proposed model, where analytical results are compared against solutions that are obtained using the finite element method (FEM). In the second example, we emulate the response of inhomogeneous electro-active bodies that are composed of a soft matrix filled with a stiff inclusion having a spherical shape, where the influence of the inclusion volume fraction on the simulated response is investigated, for both the density-dependent and the nonlinear deformationdependent electro-mechanical models as shown in Sections 3.3 and 3.4, respectively. As a third example, we illustrate the influence of ellipsoidal inclusions with different aspect ratios on the electro-mechanical behavior of the considered structure. 


\subsection{Analytical solution of electro-mechanical Neo-Hookean and extended tube models}

We simulate the electro-mechanical response of a homogeneous cubic structure using both analytical and numerical approaches. Regarding the electro-mechanical coupling, we adopt the nonlinear electro-elastic model as shown in Section 3.4. In our analysis, we drive the structure by applying an electric field and estimate the resulting stretch along the direction of the applied electric field. Regarding the analytical solution of an incompressible material with homogeneous state of deformation and one-dimensional electric field, we introduce the right Cauchy-Green deformation tensor $\boldsymbol{C}$ and the electric field $\mathbb{E}$ at the reference configuration as

$\boldsymbol{C}=\left[\begin{array}{ccc}\lambda^{-1} & 0 & 0 \\ 0 & \lambda^{-1} & 0 \\ 0 & 0 & \lambda^{2}\end{array}\right], \quad \mathbb{E}=\left[\begin{array}{l}0 \\ 0 \\ \mathrm{E}\end{array}\right]$,

with the stretch $\lambda$ along the direction of the applied electric field E. We define a normalized energy-enthalpy density function $\hat{\Psi}_{N}^{t o t}$ as

$\hat{\Psi}_{N}^{\text {tot }}(\lambda, \mathrm{E})=\Psi_{N}^{\text {hypel }}(\lambda)+\bar{\Psi}_{N}^{\text {coup }}(\lambda, \mathrm{E})$.

For an incompressible Neo-Hookean material subjected to a homogeneous deformation, the hyperelastic energy density function can be written in its normalized form as

$\Psi_{N}^{\text {hypel }}(\lambda)=\frac{1}{2}\left(\lambda^{2}+2 \lambda^{-1}-3\right)$.

Considering the extended tube model as described in Eq. 23, we introduce its simplified and normalized counterpart for incompressible materials with homogeneous boundary conditions and write $\Psi_{N}^{\text {hypel }}$ as

$$
\begin{aligned}
& \Psi_{N}^{\text {hypel }}(\lambda)=W_{N}^{c}(\lambda)+L_{N}^{e}(\lambda), \\
& W_{N}^{c}(\lambda)=\frac{R_{c}}{2}\left[\frac{\left(1-\delta^{2}\right)\left(\lambda^{2}+2 \lambda^{-1}-3\right)}{1-\delta^{2}\left(\lambda^{2}+2 \lambda^{-1}-3\right)}+\ln \left(1-\delta^{2}\left(\lambda^{2}+2 \lambda^{-1}-3\right)\right)\right], \\
& L_{N}^{e}(\lambda)=2 \frac{R_{e}}{\beta^{2}}\left(2 \lambda^{\beta / 2}+\lambda^{-\beta}-3\right),
\end{aligned}
$$

with the ratios $R_{c}=G_{c} / G[-], R_{e}=G_{e} / G$ [-], and the total shear modulus $G=G_{c}+G_{e}$. Furthermore, the coupled enthalpy density function as defined by Eqs. 28 and 29 can be rewritten in a normalized and reduced form for incompressible materials with homogeneous stress state as

$\bar{\Psi}_{N}^{\text {coup }}(\lambda, \overline{\mathrm{E}})=R_{1} \overline{\mathrm{E}}^{2}+R_{2} \lambda^{2} \overline{\mathrm{E}}^{2}$,

where $R_{1}=c_{1} / \epsilon_{0}[-], R_{2}=c_{2} / \epsilon_{0}[-]$, and $\overline{\mathrm{E}}=\mathrm{E} / \sqrt{G / \epsilon_{0}}$ [-]. As the coupled electro-mechanical problem under consideration is stress-free where no external mechanical forces are applied, the one-dimensional total first Piola-Kirchhoff stress $P$ vanishes and is introduced as

$$
\begin{aligned}
& P=P^{\text {hypel }}+P^{\text {coup }}=0, \\
& P^{\text {hypel }}=\partial_{\lambda} \Psi_{N}^{\text {hypel }}(\lambda), \\
& P^{\text {coup }}=\partial_{\lambda} \bar{\Psi}_{N}^{\text {coup }}(\lambda, \overline{\mathrm{E}}),
\end{aligned}
$$

with the hyperelastic $P^{\text {hypel }}$ and the coupled $P^{\text {coup }}$ stresses. Note that $P^{\text {coup }}$ is referred to as the Maxwell stress in some contributions; see for example [9]. We substitute (51) into (52.3) to yield the specific form of the coupled stress

$P^{\text {coup }}=2 R_{2} \lambda \overline{\mathrm{E}}^{2}$. 
Considering the Neo-Hookean material model, the hyperelastic part of the stresses $P^{\text {hypel }}$ can be computed through inserting (49) in Eq. 52.2, which yields

$$
P^{\text {hypel }}=\lambda^{2}-\lambda^{-1} \text {. }
$$

In order to perform the analytical solution of the coupled Neo-Hookean model, we substitute (53) and (54) into (52.1), which leads to a closed-form expression for the stretch $\lambda$ in terms of the normalized electric field $\overline{\mathrm{E}}$, where

$\lambda=\sqrt[3]{\frac{1}{2 R_{2} \overline{\mathrm{E}}^{2}+1}}$.

Regarding the solution of the extended tube model, we use Eqs. 50 and 52.2 to express the hyper-elastic portion of the stresses as

$$
\begin{aligned}
& P^{\text {hypel }}=P^{W}+P^{L}, \\
& P^{W}=R_{c}\left[\frac{\left(\lambda^{2}-\lambda^{-1}\right)\left(1-2 \delta^{2}+\delta^{4}\left(\lambda^{2}+2 \lambda^{-1}-3\right)\right)}{\left(1-\delta^{2}\left(\lambda^{2}+2 \lambda^{-1}-3\right)\right)^{2}}\right], \\
& P^{L}=2 \frac{R_{e}}{\beta}\left(\lambda^{(\beta / 2-1)}-\lambda^{-(\beta+1)}\right) .
\end{aligned}
$$

Subsequently, we insert (53) and (56) into (52.1) to find the analytical solution of the electro-mechanical extended tube model in terms of the stretch $\lambda$. However, due to the complexity of Eq. 56, we solve for $\lambda$ by using the Newton-Raphson method, in contrast to the Neo-Hookean counterpart, where a closed form of the solution can be easily obtained. Regarding the material parameters for the extended tube model, we use $\beta=1.0[-], R_{c}=0.9375[-], R_{e}=0.0625[-]$, and two different values for the limited chain extensibility parameter $\delta=0.20[-]$ and $\delta=0.60[-]$. Moreover, we set the electro-mechanical coupling parameter as $R_{2}=250$ [-].

Regarding the finite element analyses, we specify the bulk modulus parameter $\kappa$ to emulate quasi-incompressible behavior of the material, such that the initial Poisson ratio yields $v_{0}=0.499$ [-]. We discretize a cubic structure with dimensions of $L \times L \times L$ using $(m \times m \times m) \times 6$ tetrahedral finite elements with $m=7$. In order to verify the insensitivity of the finite element formulation toward mesh distortion, we randomly displace three nodes on each of the six inner surfaces of the discretized cubic structure. For further illustration, Fig. 2 shows the distortion at two different inner surfaces of the body. Moreover, the whole discretized structure is demonstrated in Fig. 2c. Figure 3 visualizes a finite element simulation of the considered problem, where a voltage difference between the two surfaces of the cube induces a one-dimensional electric field. As it is demonstrated in Fig. 4, the analytical and the numerical solutions of the considered problem fit together to

(a)

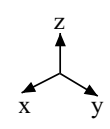

(b)

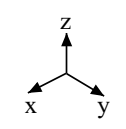

(c)<smiles>CC(C)C(C)C(C)(C)C</smiles>
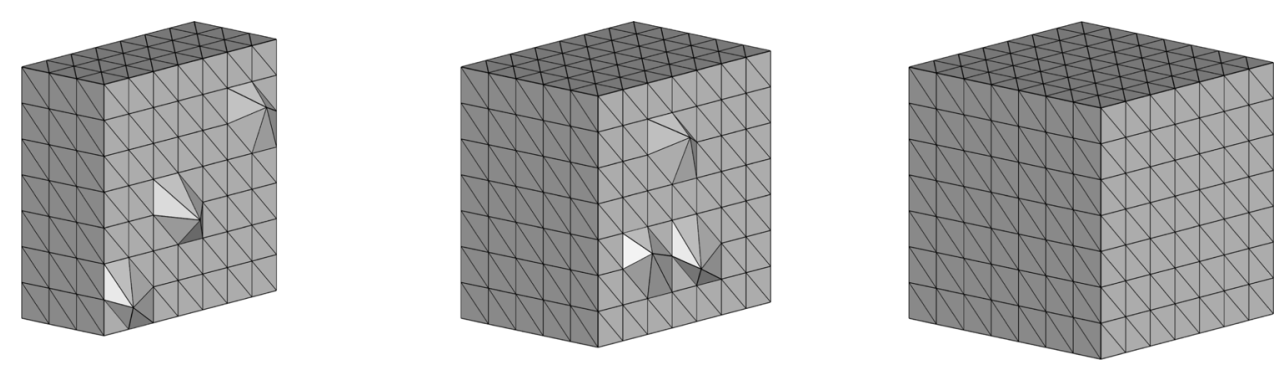

Fig. 2 Finite element discretization of the cubic structure with cross sections along the y-axis at (a) $3 m / 7$ and (b) $5 m / 7$ where $m=7$ is the number of finite elements along the length $L$, and (c) a view of the whole structure 
(a)

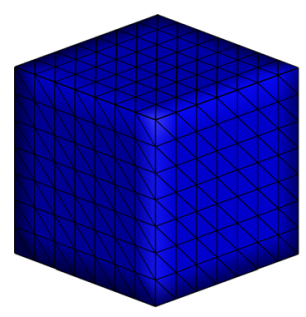

(b)

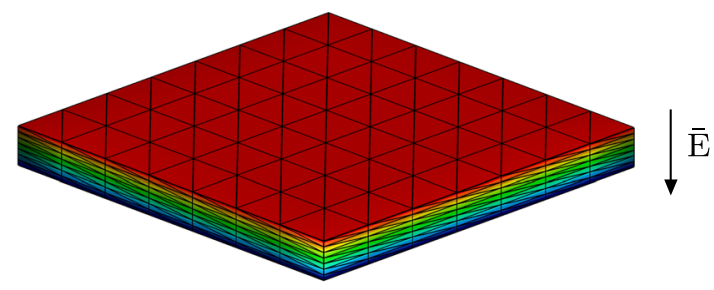

$$
\phi^{\min }=\phi[\mathrm{V}]
$$

Fig. 3 Finite element simulation of the electro-mechanical cubic structure at (a) undeformed and (b) deformed states. The contour shows the electric potential $\phi$ in Volts. The arrow indicates the direction of the applied electric field $\overline{\mathrm{E}}$

a high extent, for both the electro-mechanical Neo-Hookean and extended tube models. Furthermore, the finite element simulations are performed using both undistorted and distorted meshes. The simulation results are identical for both meshes.

\subsection{Composites with spherical inclusions}

We study the electro-mechanical response of inhomogeneous bodies consisting of a soft matrix and a stiff spherical inclusion with different volume fractions $f$ of the inclusion. In order to express the global electric response of the considered

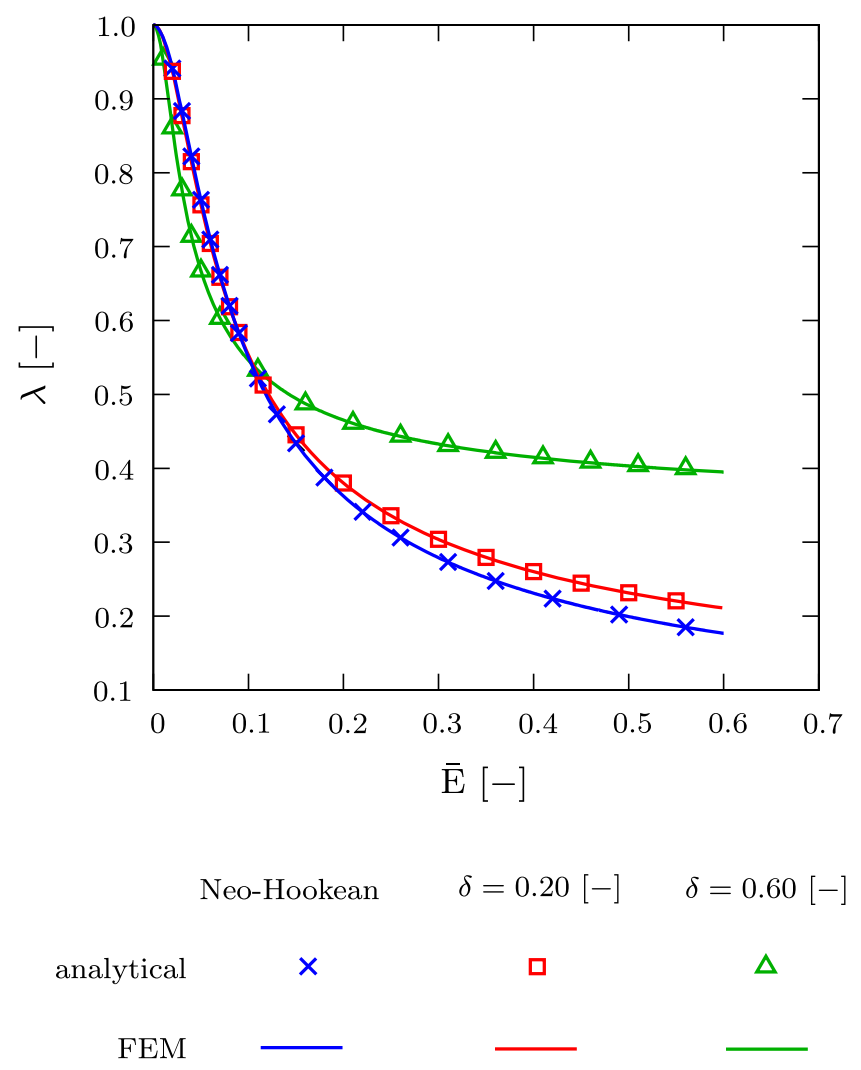

Fig. 4 Analytical and numerical results for the electro-mechanical cubic structure 
inhomogeneous structure, we assess the relation between the normalized and averaged electric field $\tilde{\tilde{\mathbb{E}}}[-]$ and electric displacement $\tilde{\overline{\mathbb{D}}}[-]$, where

$\tilde{\overline{\mathbb{E}}}=\frac{1}{V \sqrt{G_{0} / \epsilon_{0}}} \int_{\mathcal{B}} \mathbb{E} d V \quad$ and $\quad \tilde{\overline{\mathbb{D}}}=\frac{1}{V \sqrt{G_{0} \epsilon_{0}}} \int_{\mathcal{B}} \mathbb{D} d V$

with the total reference volume $V$ of the composite. Moreover, in order to evaluate the actuation or the intensity of the electro-mechanical coupling, we study the relation between $\tilde{\overline{\mathbb{E}}}[-]$ and the averaged deformation gradient $\tilde{\boldsymbol{F}}$ of the whole structure using

$\tilde{\boldsymbol{F}}=\frac{1}{V} \int_{\mathcal{B}} \boldsymbol{F} d V$

We perform finite element simulations of the structure considering varying volume fractions $f=\{0,5,10,15$, and $20 \%\}$. For describing the purely hyperelastic response of the soft matrix, we use the extended tube model and assign a total shear modulus $G_{0}$, with $G_{c}=0.9375 G_{0}, G_{e}=0.0625 G_{0}, \delta=0.20[-], \beta=1.0[-]$, and $\nu_{0}=0.499$ [-]. Moreover, the Neo-Hookean material parameters of the stiff inclusion read $G=100 G_{0}$ and $\nu_{0}=0.40$ [-]. Regarding the electroelastic coupled response, we utilize the dielectrical quasi-linear coupled ansatz as it is demonstrated in Section 3.3 and the model of nonlinear electro-elasticity with deformation-dependent electric properties as it is outlined in Section 3.4. For the dielectrical quasi-linear coupled ansatz (Section 3.3), we consider the parameter $\epsilon=\epsilon_{0}$ for the soft matrix and $\epsilon=1000 \epsilon_{0}$ with regard to the electro-mechanical coupling in the inclusion. Regarding the deformation-dependent electro-mechanical model (Section 3.4), the material parameters are chosen such that they denote base electric and coupled properties of the material which are equivalent to the assumed properties using the parameters $\epsilon=\epsilon_{0}$ with respect to matrix and $\epsilon=1000 \epsilon_{0}$ with regard to the inclusion, for the only density-dependent counterpart (Section 3.3). Therefore, we utilize the parameters $c_{1}=-\epsilon_{0}$ and $c_{2}=0.5 \epsilon_{0}$ to express the coupled response in the matrix and we set the parameters as $c_{1}=-1000 \epsilon_{0}$ and $c_{2}=500 \epsilon_{0}$ to describe the electro-mechanical interaction within the stiff inclusion. The chosen parameters indicate that the inclusion is stiffer and has higher electric permittivity than the matrix. The electro-mechanical analysis is driven by applying an electric potential difference. Moreover, the mechanical boundary conditions are assigned such that the boundaries of the full composite are unconstrained. As the applied boundary conditions are symmetric, we simulate only one-eighth of the whole structure. Note that similar mechanical boundary conditions are adopted in [9].

The structures are discretized using tetrahedral finite elements with geometry adaptive sizing of the elements. The minimum and the maximum sizes of the used elements are the same for all composites with different volume fractions of the spherical inclusion. Finite element meshes of the composite with $f=5 \%$ and $f=20 \%$ are illustrated in Fig. 5, where the whole inclusion and one-half of the matrix are shown. Regarding the analysis results, Fig. 6 depicts the deformed state of the inhomogeneous structures with varying volume fractions of the spherical inclusion. It can be observed that the distribution of the electric voltage is disturbed due to the existence of the high electric permittive inclusion. Moreover, the electric fields tend to zero within the inclusion compared to the fields within the region of the soft matrix. See the distribution of the

(a)

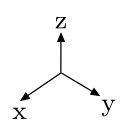

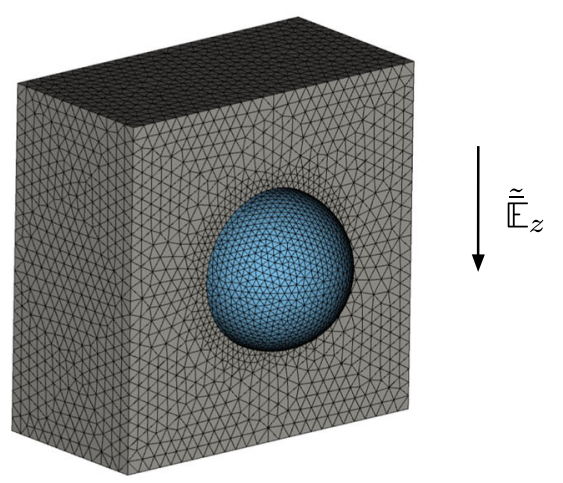

(b)

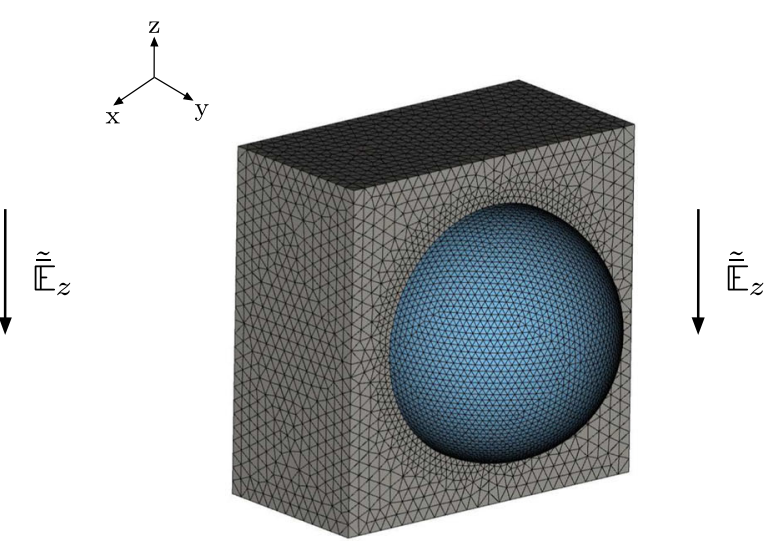

Fig. 5 Finite element mesh of one-half of the soft matrix and the whole spherical inclusion with a volume fraction (a) $f=5 \%$ and (b) $f=20 \%$. The arrows show the direction of the global electric field $\tilde{\mathbb{E}}_{z}$ with positive sign 
Fig. 6 Finite element

simulations of inhomogeneous

material structures with a

volume fraction of the spherical inclusion (a) $f=5 \%$,

(b) $f=10 \%$, (c) $f=15 \%$, and

(d) $f=20 \%$. The contour

shows the electric potential $\phi$ in

Volts and the vectors

demonstrate the orientation of

the electric field $\mathbb{E}$. The analyses

are performed using the

nonlinear electro-mechanical

description as in Section 3.4 (a)

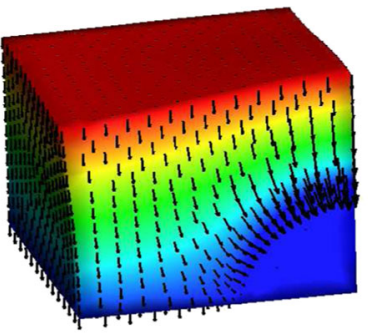

(c)

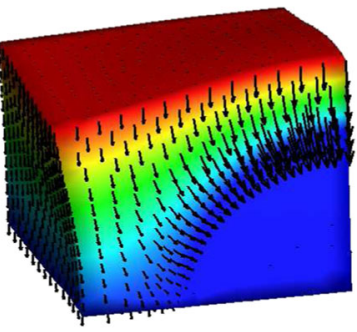

(b)

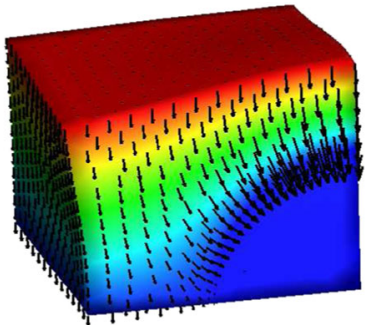

(d)

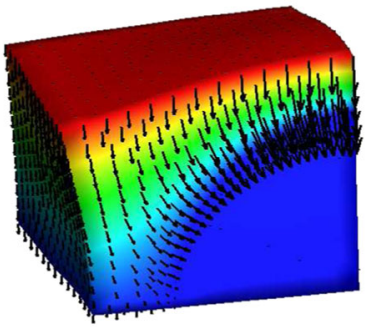

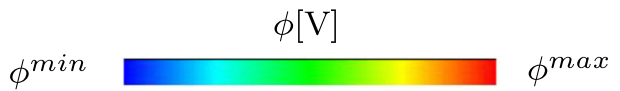

electric potential $\phi$ and the electric field $\mathbb{E}$ in Fig. 6. In [9], the same coupled model as in Section 3.3 is utilized along with a hyperelastic Neo-Hookean material model to perform stability analyses of similar configurations as the ones presented in this section. It is discussed in [9] that an analysis of the structural configuration under consideration using the coupled ansatz as in Section 3.3, with the simulation being driven by an electric field $\tilde{\mathbb{E}}_{z}$, allows for performing the analysis up to the point where electro-mechanical instability occurs. Therefore, the authors have suggested that in order to trace the response after the occurrence of instability, the electro-mechanical response of the structure should be controlled by applying an electric displacement $\tilde{\overline{\mathbb{D}}}_{z}[9]$. Moreover, they [9] have demonstrated that for the considered configurations with the assumption that

(a)

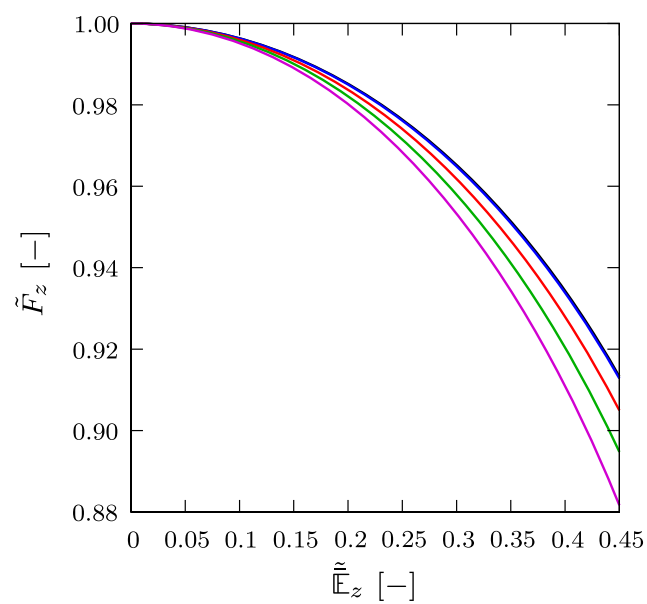

$f=0 \%-$ (b)
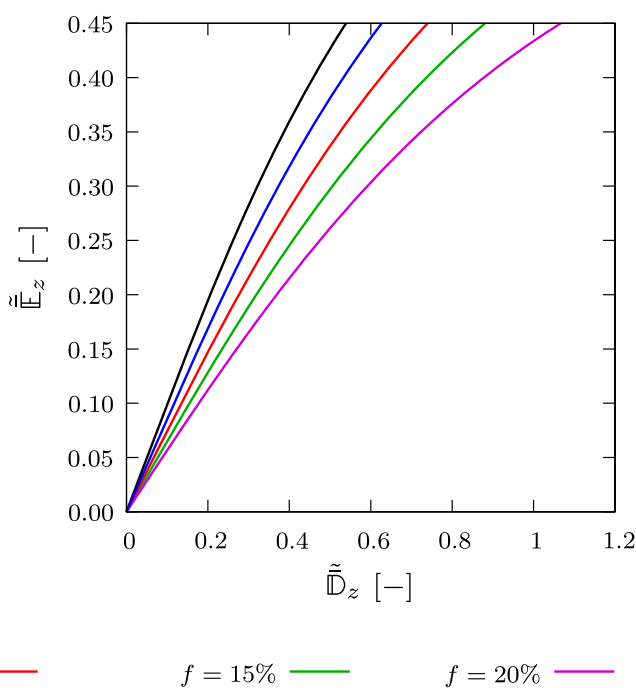

Fig.7 Plot of (a) averaged deformation $\tilde{F}_{z}$ and $(\mathbf{b})$ averaged and normalized electric displacement $\tilde{\overline{\mathbb{D}}}_{z}[-]$ versus averaged and normalized electric field $\tilde{\tilde{\mathbb{E}}}_{z}[-]$, for different fractions $f$ of the spherical inclusion. Analyses are performed using the dielectrical quasi-linear electro-mechanical description as in Section 3.3 
(a)

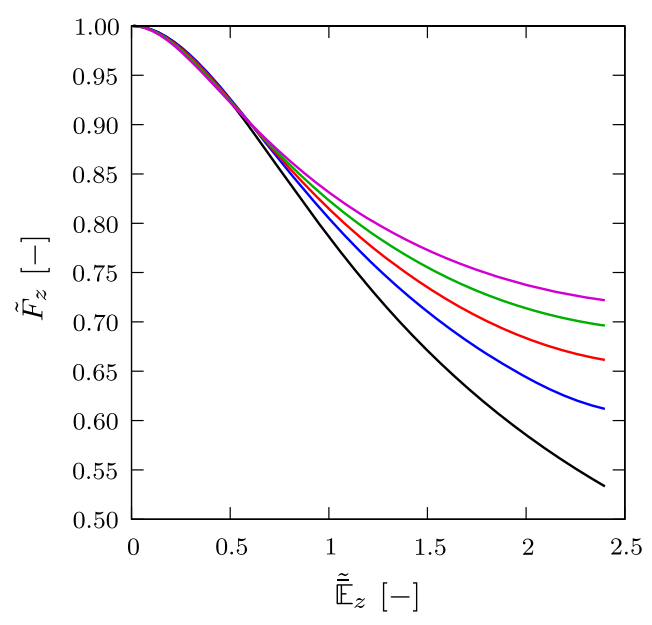

(b)

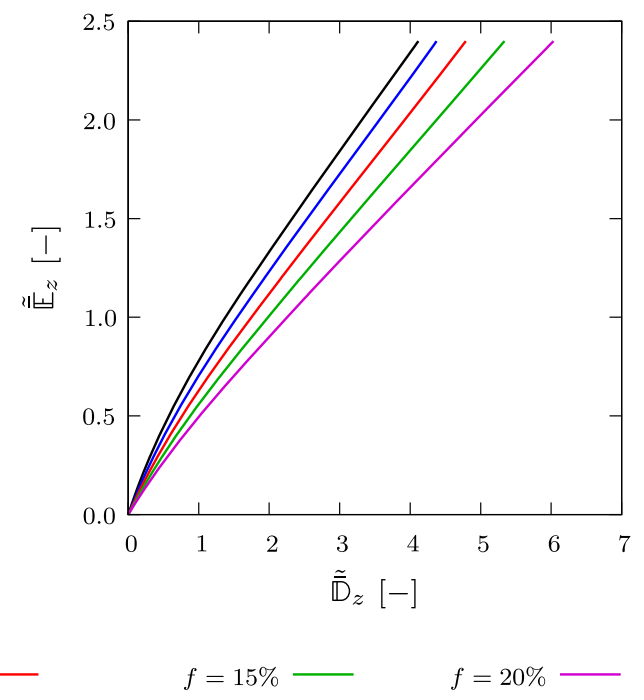

Fig. 8 Plot of (a) averaged deformation $\tilde{F}_{z}$ and (b) averaged and normalized electric displacement $\tilde{\overline{\mathbb{D}}}_{z}[-]$ versus averaged and normalized electric field $\tilde{\overline{\mathbb{E}}}_{z}[-]$ with relatively high levels for different fractions $f$ of the spherical inclusion. Analyses are performed using the nonlinear electro-mechanical description as in Section 3.4

the material exhibits a dielectrical quasi-linear electro-mechanical behavior as described in Section 3.3, the instability point is reached for levels of the normalized electric field $\tilde{\overline{\mathbb{E}}}_{z}<1$. In this contribution, we use the dielectrical quasi-linear model as shown in Section 3.3 and the nonlinear electro-elastic description as expressed in Section 3.4, both coupled to the extended tube model to perform our study. Moreover, as stability analysis is not our topic, we drive the numerical simulations by applying an electric field $\tilde{\overline{\mathbb{E}}}_{z}$ within a range $\tilde{\overline{\mathbb{E}}}_{z} \leq 0.45$, before all the considered structures become electro-mechanically unstable. The influence of the inclusion size with respect to the soft matrix on the total actuation intensity and the global electric response of the composite is illustrated in Fig. 7. We can observe from the $\tilde{\overline{\mathbb{E}}}_{z}-\tilde{F}_{z}$ relation as it is shown in Fig. 7a that the actuation capability gets improved as the relative size of the inclusion is increased in terms of $f$. Moreover, the

(a)

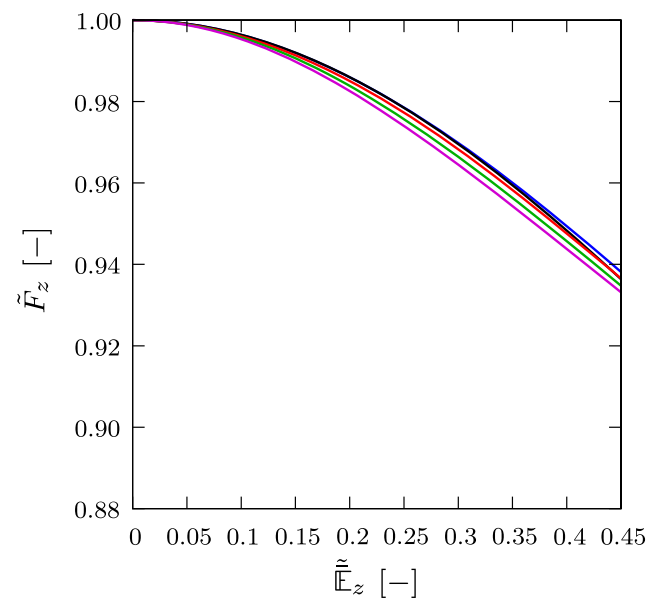

$f=0 \%$ (b)

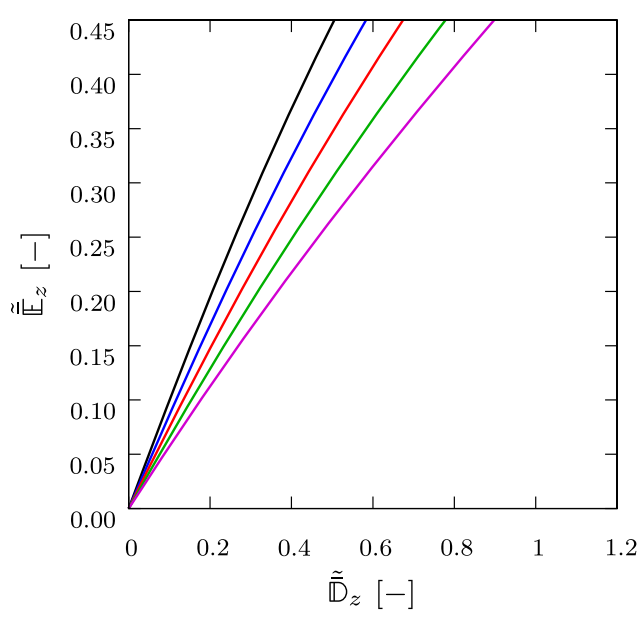

$f=15 \%-f=20 \%-$

Fig. 9 Plot of (a) averaged deformation $\tilde{F}_{z}$ and (b) averaged and normalized electric displacement $\tilde{\overline{\mathbb{D}}}_{z}[-]$ versus averaged and normalized electric field $\tilde{\tilde{\mathbb{E}}}_{z}[-]$ with relatively low levels for different fractions $f$ of the spherical inclusion. Analyses are performed using the nonlinear electro-mechanical description as in Section 3.4 
(a)<smiles>[Y]C([3H])[3H]</smiles>

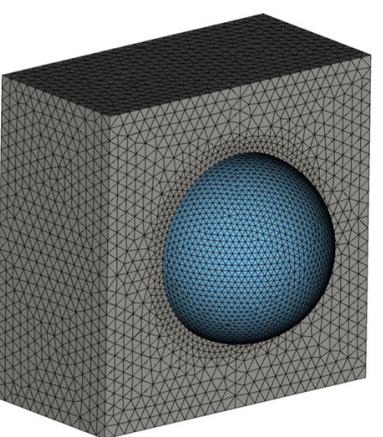

(b)

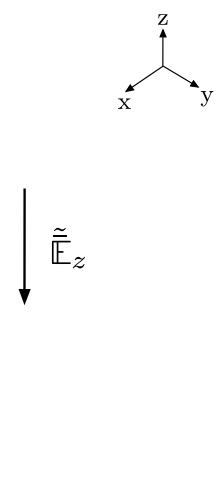

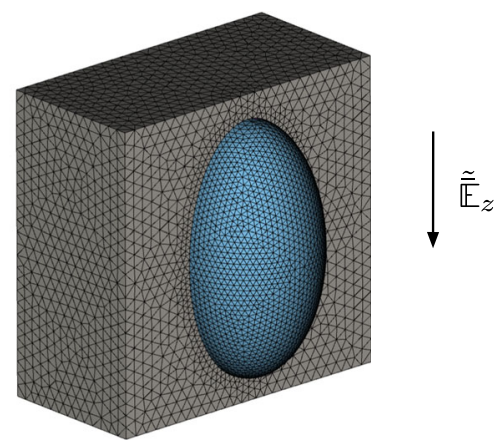

Fig. 10 Finite element mesh of one-half of the soft matrix and the whole inclusion with aspect ratio (a) $r=1.00[-]$ and $(\mathbf{b}) r=1.75[-]$, where $f=10 \%$. The arrows show the direction of the global electric field $\tilde{\mathbb{E}}_{z}$ with positive sign

results of the electric response as shown in Fig. 7b indicate that as $f$ increases, larger electric displacements $\tilde{\overline{\mathbb{D}}}_{z}$ result in response to the same applied $\tilde{\overline{\mathbb{E}}}_{z}$. Figure 8 illustrates the results of the simulations for composites assuming that the electroelastic coupling is based on the description as in Section 3.4 for a relatively large range of electric fields (i.e., $\tilde{\overline{\mathbb{E}}}_{z}>1$ ). Regarding the simulation of the composites assuming nonlinear dependency of the electric properties on deformation, electro-mechanical instability does not occur at least at low levels of the electric field (i.e., $\tilde{\overline{\mathbb{E}}}_{z}<1$ ), which is not the case for the earlier considered dielectrical quasi-linear materials. From a pure engineering point of view, instability at relatively low levels of electric loading does not take place in electrically nonlinear materials because the material adapts its electric and coupled properties in response to the deformation, which leads to overpassing the instability point. Figure 8a depicts that at low levels of the electric field $\tilde{\overline{\mathbb{E}}}_{z}$, the actuation intensity is in general enhanced as $f$ is set larger. However, at higher levels of the electric field, the actuation behavior is diminished as the relative size of the inclusion increases. On the other hand, the results of the electric response as shown in Fig. $8 \mathrm{~b}$ imply that as $f$ increases, larger electric displacements $\tilde{\overline{\mathbb{D}}}_{z}$ result with regard to the same applied $\tilde{\overline{\mathbb{E}}}_{z}$. In order to compare the results of the dielectrical quasi-linear material and its electrically

(a)

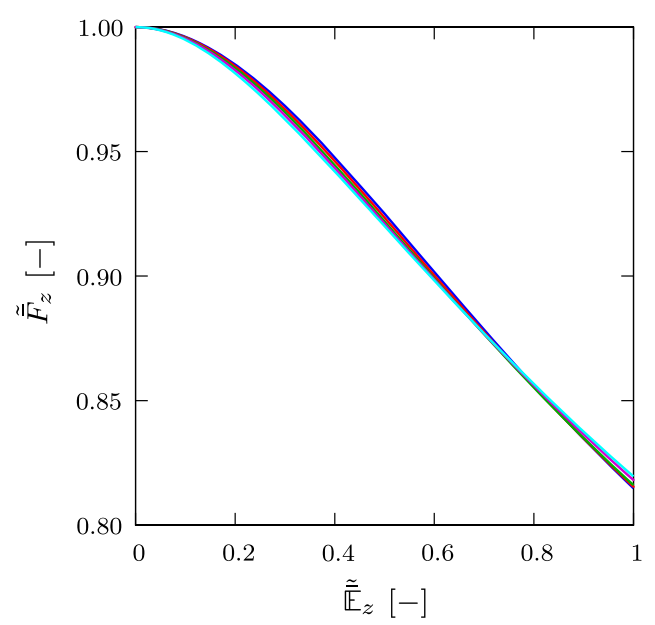

(b)

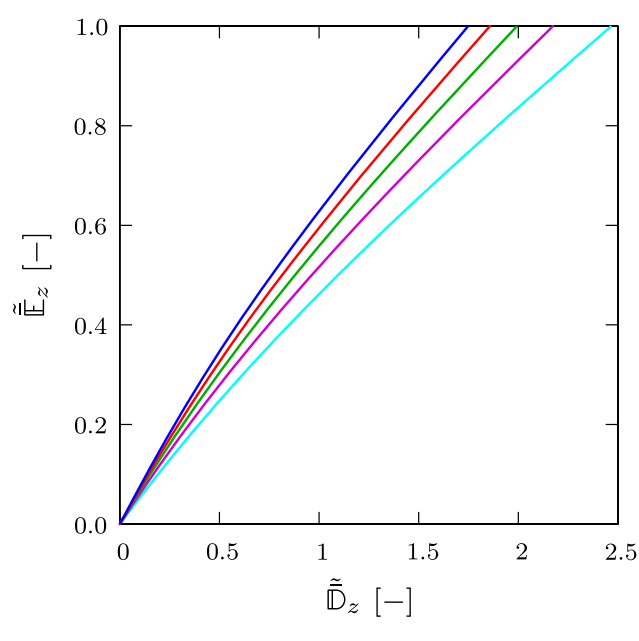

$$
r=1.00[-]-\quad r=1.25[-]-\quad r=1.50[-]-\quad r=1.75[-]-\quad r=2.00[-]-
$$

Fig. 11 Plot of (a) averaged deformation $\tilde{F}_{z}$ and $(\mathbf{b})$ averaged and normalized electric displacement $\tilde{\overline{\mathbb{D}}}_{z}$ versus averaged and normalized electric field $\tilde{\tilde{\mathbb{E}}}_{z}$, for different aspect ratios $r$ of the inclusion, where $f=10 \%$. Analyses are performed using the nonlinear electro-mechanical description as in Section 3.4 


\section{Fig. 12 Finite element}

simulations of inhomogeneous structures with aspect ratio of the inclusion (a) $r=1.00[-]$, (b) $r=1.25[-]$, (c) $r=1.50$ [-] and (d) $r=1.75$ [-] where $f=10 \%$. The contour shows the electric potential $\phi$ in Volts and the vectors demonstrate the orientation of the electric field $\mathbb{E}$. Analyses are performed using the nonlinear electro-mechanical description as in Section 3.4 (a)

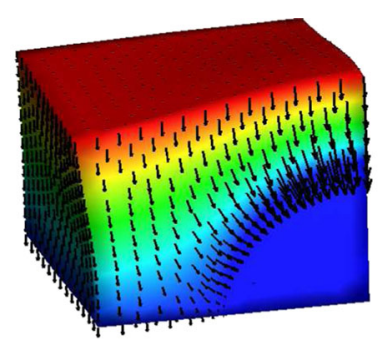

(c)

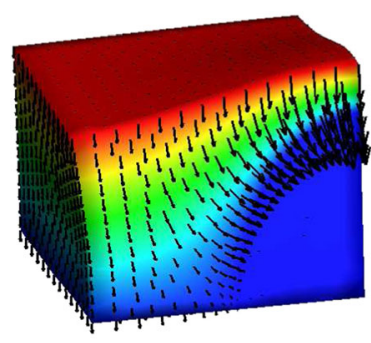

(b)

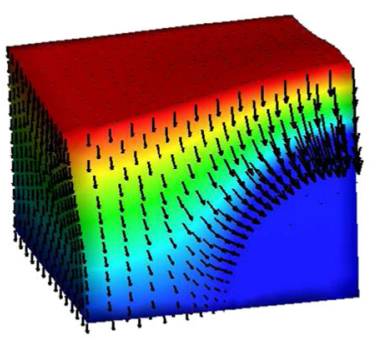

(d)

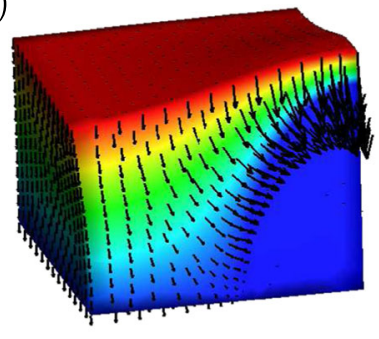

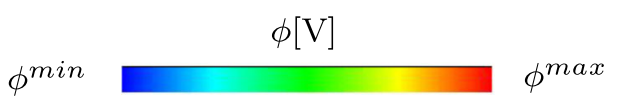

nonlinear counterpart, we extract the results of Fig. 8, in which the maximum applied electric field is $\tilde{\overline{\mathbb{E}}}_{z}=0.45[-]$ and demonstrate them in Fig. 9. We can notice from Fig. 9a that a general trend of actuation improvement is observed, which is caused by increasing the volume fraction of the inclusion. However, comparing Figs. 7a to 9a leads to the conclusion that dielectrically quasi-linear materials are more sensitive toward varying volume fractions of the spherical inclusion, compared to their counterparts with nonlinearly deformation-dependent electro-mechanical properties.

\subsection{Composites with ellipsoidal inclusions}

We investigate the influence of the aspect ratio $r$ of ellipsoidal inclusions on the electro-mechanical behavior of inhomogeneous bodies, utilizing the electro-mechanical model with deformation-dependent electric properties; see Section 3.4. We consider a fixed volume fraction $f=10 \%$ and varying aspect ratios $r=\{1.00,1.25,1.50,1.75$, and 2.00 [-]\}. Using a geometry adaptive meshing scheme, all composites with different aspect ratios of the ellipsoidal inclusion are discretized using tetrahedral finite elements. Moreover, we apply the same minimum and maximum sizes of the finite elements for all the considered examples. Figure 10 depicts the finite element meshes of composites with $r=1.00[-]$ and $r=1.75[-]$.

Regarding the results of the analyses, Fig. 11a shows that at low electric field levels, the actuation is slightly improved as the aspect ratio $r$ increases. However, the actuation intensity at higher levels of the electric field is reduced as $r$ is set larger. Nevertheless, relatively, no significant influence of changing the aspect ratio on the actuation behavior is found. Furthermore, we can notice from Fig. 11b that higher electric displacements are obtained for the same electric field as the aspect ratio of the inclusion $r$ is larger. The finite element simulations for composites with $r=\{1.00,1.25,1.50$, and 1.75 $[-]\}$ are shown in Fig. 12.

\section{Conclusion}

In the presented work, we have proposed a constitutive material description and the associated finite element implementation, which are together adequate for the simulation of the coupled electro-mechanical response of inhomogeneous EAP at large deformations. The modelling framework includes the hyperelastic extended tube model coupled to two distinct electromechanical mathematical descriptions. The capabilities of our suggested framework are demonstrated through analytical 
and numerical examples, with emphasis on heterogeneous bodies. In future contributions, we aim to employ the presented model to study material and structural electro-mechanical instabilities in EAP.

Funding Open Access funding enabled and organized by Projekt DEAL. The DFG research project 380321452/GRK2430 is supported by the Deutsche Forschungsgemeinschaft (DFG, German Research Foundation). The financial support is gratefully acknowledged.

Open Access This article is licensed under a Creative Commons Attribution 4.0 International License, which permits use, sharing, adaptation, distribution and reproduction in any medium or format, as long as you give appropriate credit to the original author(s) and the source, provide a link to the Creative Commons licence, and indicate if changes were made. The images or other third party material in this article are included in the article's Creative Commons licence, unless indicated otherwise in a credit line to the material. If material is not included in the article's Creative Commons licence and your intended use is not permitted by statutory regulation or exceeds the permitted use, you will need to obtain permission directly from the copyright holder. To view a copy of this licence, visit http://creativecommonshorg/licenses/by/4.0/.

\section{References}

1. Pelrine, R., Kornbluh, R., Pei, Q., Joseph, J.: High-speed electrically actuated elastomers with strain greater than $100 \%$. Science $\mathbf{2 8 7}$, 836839 (2000)

2. Bar-Cohen, Y.: Electro-active polymers: current capabilities and challenges. Proceedings of SPIE 4695, Smart Structures and Materials Symposium, Electro-active Polymer Actuators and Devices Conference, San Diego (2002)

3. Pfeil, S., Katzer, K., Kanan, A., Mersch, J., Zimmermann, M., Kaliske, M., Gerlach, G.: A biomimetic fish fin-like robot based on textile reinforced silicone. Micromachines 11, 1-16 (2020)

4. Shian, S., Bertoldi, K., Clarke, D.: Dielectric elastomer based "grippers" for soft robotics. Adv Mater 27, 6814-6819 (2015)

5. Xing, Z., Zhang, J., McCoul, D., Cui, Y., Sun, L., Zhao, J.: A super-lightweight and soft manipulator driven by dielectric elastomers. Soft Robot. 7, 1-9 (2020)

6. Pelrine, R., Kornbluh, R., Joseph, J.: Electrostriction of polymer dielectrics with compliant electrodes as a means of actuation. Sens. Actuators A Phys. 64, 77-85 (1998)

7. Wissler, M., Mazza, E.: Electromechanical coupling in dielectricelastomer actuators. Sens. Actuators A Phys. 138, 384-393 (2007)

8. Miehe, C., Vallicotti, D., Teichtmeister, S.: Homogenization and multiscale stability analysis in finite magneto-electro-elasticity. Application to soft matter EE, ME and MEE composites. Comput. Methods Appl. Mech. Eng. 300, 294-346 (2015)

9. Miehe, C., Vallicotti, D., Zäh, D.: Computational structural and material stability analysis in finite electro-elasto-statics of electro-active materials. Int. J. Numer. Methods Eng. 102, 1605-1637 (2015)

10. Ponte Castañeda, P., Siboni, M.H.: A finite-strain constitutive theory for electro-active polymer composites via homogenization. Int. J. Non-linear Mech. 47, 293-306 (2012)

11. Tian, L., Tevet-Deree, L., deBotton, G., Bhattacharya, K.: Dielectric elastomer composites. J. Mech. Phys. Solids 60, 181-198 (2012)

12. Böse, H., Uhl, D., Flittner, K., Sclaak, H.: Dielectric elastomer actuator with enhanced permittivity and strain. Proc. SPIE - Int. Soc. Opt. Eng. 7976, 79762J (2011)

13. Risse, S., Kussmaul, B., Kürger, H., Kofod, G.: Synergistic improvement of actuation properties with compatibilized high permittivity filler. Adv Funct Mater 22, 3958-3962 (2012)

14. Stoyanov, H., Kollosche, M., Risse, S., McCarthy, D.N., Kofod, G.: Elastic block copolymer nanocomposites with controlled interfacial interactions for artificial muscles with direct voltage control. Soft Matter 7, 194-202 (2011)

15. Eringen, A.: On the foundations of electroelastostatics. Int. J. Eng. Sci. 1, 127-153 (1963)

16. Lax, M., Nelson, D.F.: Linear and nonlinear electrodynamics in elastic anisotropic dielectric. Phys. Rev. B 4, 3694-3731 (1971)

17. Maugin, G.A.: Continuum mechanics of electromagnetic solids, vol. 33. North Holland Series in Applied Mathematics and Mechanics, North Holland (1988)

18. Maugin, G.A.: On modelling electromagnetomechanical interactions in deformable solids. Int. J. Adv. Eng. Sci. Appl. Math. 1, 25-32 (2009)

19. Dorfmann, A., Ogden, R.W.: Nonlinear electroelasticity. Acta Mech. 174, 167-183 (2005)

20. Dorfmann, A., Ogden, R.W.: Nonlinear electroelastic deformations. J. Elast. 82, 99-127 (2006)

21. Dorfmann, L., Ogden, R.W.: Nonlinear electroelasticity: material properties, continuum theory and applications. R. Soc. Publish. 56, 1-34 (2017)

22. Jiménez, S., McMeeking, R.: A constitutive law for dielectric elastomers subject to high levels of stretch during combined electrostatic and mechanical loading: Elastomer stiffening and deformation dependent dielectric permittivity. Int. J. Non-linear Mech. 87, 125-136 (2016)

23. McMeeking, R., Landis, C.: Electrostatic forces and stored energy for deformable dielectric materials. J. Appl. Mech. 72, 518-590 (2005)

24. Vu, D.K., Steinmann, P., Possart, G.: Numerical modelling of non-linear electroelasticity. Int. J. Numer. Methods Eng. 70, 685-704 (2007)

25. Zäh, D., Miehe, C.: Multiplicative electro-elasticity of electroactive polymers accounting for micromechanically-based network models. Comput. Methods Appl. Mech. Eng. 286, 394-421 (2015)

26. Pelteret, J.P., Davydov, D., McBride, A., Vu, D.K., Steinmann, P.: Computational electro-elasticity and magneto-elasticity for quasiincompressible media immersed in free space. Int. J. Numer. Methods Eng. 108, 1307-1342 (2016)

27. Arruda, E.M., Boyce, M.C.: A three-dimensional model for the large stretch behavior of rubber elastic materials. J. Mech. Phys. Solids 41, 389-412 (1993)

28. Mehnert, M., Hossain, M., Steinmann, P.: Experimental and numerical investigations of the electro-viscoelastic behavior of VHB $4905^{T M}$. Eur. J. Mech. / A Solids 77, 1-9 (2019)

29. Bishara, D., Jabareen, M.: A reduced mixed finite element formulation for modeling the viscoelastic response of electroactive polymers at finite deformation. Math. Mech. Solids 24, 1578-1610 (2019) 
30. Dorfmann, L., Ogden, R.W.: Instabilities of soft dielectrics. R. Soc. Publish. 377, 1-35 (2019)

31. Su, Y., Broderick, H.C., Chen, W., Destrade, M.: Wrinkles in soft dielectric plates. J. Mech. Phys. Solids 119, 298-318 (2018)

32. Kaliske, M., Heinrich, G.: An extended tube-model for rubber elasticity: Statistical-mechanical theory and finite element implementation. Rubber Chem. Technol. 72, 602-632 (1999)

33. Vogel, F., Göktepe, S., Steinmann, P., Kuhl, E.: Modeling and simulation of viscous electro-active polymers. Eur. J. Mech. Solids 48, 112-128 (2014)

34. Bustamante, R.: A variational formulation for a boundary value problem considering an electro-sensitive elastomer interacting with two bodies. Mech. Res. Commun. 36, 791-795 (2009)

35. Behnke, R., Kaliske, M.: The extended non-affine tube model for crosslinked polymer networks: physical basics, implementation, and application to thermomechanical finite element analyses. In: Stöckelhuber, K., Das, A., Klüppel, M. (eds.) Designing of Elastomer Nanocomposites: From Theory to Applications. Advances in Polymer Science, vol. 275. Springer, Cham (2016)

36. Kanan, A., Kaliske, M.: Finite element modeling of electro-viscoelasticity in fiber reinforced electro-active polymers. Int J Numer Methods Eng., 1-33. https://doi.org/10.1002/nme.6610 (2021)

Publisher's note Springer Nature remains neutral with regard to jurisdictional claims in published maps and institutional affiliations. 